\title{
IDENTIFICAÇÃO DO GRAU DE DIFICULDADE DO PROCESSO DE DESENVOLVIMENTO DE NOVOS PRODUTOS NA INDÚSTRIA DE LATICÍNIOS DO RIO GRANDE DO SUL
}

\section{The difficulty level of new product development in the dairy industry of Rio Grande do Sul state, Brazil}

Rubilene Ramos de Araújo ${ }^{1}$, Jean Philippe Palma Révillion ${ }^{1 *}$

\begin{abstract}
RESUMO
Essa pesquisa teve como objetivo identificar o grau de dificuldade, e a natureza dessas dificuldades, nas principais etapas do processo de desenvolvimento de produtos (PDP) na indústria processadora de laticínios no estado do Rio Grande do Sul. Para tanto, foi realizada uma survey junto aos gerentes, envolvidos com o processo de PDP, de 68 indústrias processadoras de laticínios com Serviço de Inspeção Federal SIF ou fiscalizados pela Coordenadoria de Inspeção de Produtos de Origem Animal - CISPOA localizadas no estado do Rio Grande do Sul. Considerando-se todas as etapas do DPD, cerca de $40 \%$ das dificuldades encontradas foram avaliadas como de natureza gerencial contra $20 \%$ de natureza financeira, $20 \%$ de natureza comercial e $20 \%$ de natureza tecnológica. Em particular, as etapas do PDP em que os tomadores de decisão das empresas consultadas encontram maior dificuldade foram relacionadas a "testes de protótipos - explorando novos ingredientes e embalagens", a "definição de fornecedores", na fase de desenvolvimento, e o "estudo do comportamento de compras e atitude dos consumidores", depois do lançamento do novo produto. Essas dificuldades podem estar relacionadas a uma mudança do perfil das inovações desenvolvidas nesse setor, de menor dependência dos fornecedores, buscando uma maior a apropriabilidade das mesmas - o que exige uma maior capacitação técnica de seus quadros e uma visão mais alinhada, e menos setorizada, entre os profissionais de "produção" e "gestão" das empresas.
\end{abstract}

Palavras-chave: processo de desenvolvimento de produto; inovação; indústria de alimentos.

1 Universidade Federal do Rio Grande do Sul (UFRGS), Programa de Pós-graduação em Agronegócios, Centro de Estudos e Pesquisas em Agronegócios (CEPAN), Av. Bento Gonçalves, 7712, Prédio da Agronomia, 1.․ Andar, 91540-000, Porto Alegre, RS, Brasil. E-mail: jeanppr@gmail.com, cepan@ufrgs.br

* Autor para correspondência 


\begin{abstract}
This research evaluates the degree of difficulty (and their nature) to perform the steps of the news products development process (PDP) in the dairy industry of Rio Grande do Sul state, Brazil. A descriptive/ quantitative survey was performed with decision makers from 68 dairy processing industries in the state of Rio Grande do Sul which are under federal or state sanitary supervision. Considering all steps of PDP, about $40 \%$ of the problems were related with management issues against $20 \%$ with financial, $20 \%$ commercial and $20 \%$ of a technological nature. In particular, the steps of the PDP in which the decision makers of the companies surveyed have more difficult were related to "testing of prototypes - exploring new ingredients and packages", "the definition of suppliers" in the development phase, and the "study of shopping behavior and consumers attitudes", after launching the new product. These difficulties may be related to a change in the profile of the innovations developed in this sector, with a lower dependence on suppliers and a greater appropriability concern - which requires greater technical skills and a more aligned vision among "production" and "management" staff.
\end{abstract}

Keywords: product development process; innovation; food industry.

\section{INTRODUÇÃO}

Essa pesquisa busca identificar o grau de dificuldade, e a natureza dessas dificuldades, nas principais etapas do PDP na indústria processadora de laticínios no estado do Rio Grande do Sul. Esse setor representa um lócus privilegiado para essa análise já que o estado concentra 162 indústrias de diferentes portes, desde multinacionais até pequenas e médias empresas, todas imersas em um ambiente institucional e competitivo favorável à inovação.

O processo de desenvolvimento de produto (PDP), segundo Ulrich; Eppinger (2000) pode ser definido como o conjunto de atividades relacionadas com o processo de desenvolvimento, produção, venda e entrega de um produto comercial elaborado para atender novas oportunidades de mercado. Em particular, a literatura sobre PDP na indústria de alimentos apresenta uma série de modelos de referência (FULLER, 1994; RUDOLPH, 1995; EARLE 1997) representações estruturadas de uma sequência de fases com etapas, atividades e tarefas genéricas associadas a cada fase.
Porém, apesar da inquestionável importância do desenvolvimento de novos produtos para a sobrevivência das empresas processadoras de alimentos (TRAIL; MEULENBERG, 2002; CABRAL, 2007), são raras as pesquisas detalhando como o PDP é, de fato, aplicado nesse setor.

Nesse contexto, a identificação e a caracterização de dificuldades no desenvolvimento de etapas específicas no PDP do setor processador de lácteos podem contribuir com a definição de quais fatores (de ordem financeira, tecnológica ou institucional) e capacidades (de natureza gerencial ou técnica) são restritivos a esse processo e quais etapas são mais afetadas por eles. Esse conhecimento pode subsidiar tanto a definição de políticas públicas voltadas ao fomento da inovação setorial como a adoção de medidas gerenciais voltadas a minimizar esses gargalos nas empresas do setor.

\section{MATERIAL E MÉTODOS}

Essa pesquisa pode ser classificada como de natureza quantitativa e descritiva, valendose de uma amostragem não probabilística e 
intencional. A pesquisa quantitativa descritiva tem como objetivo descrever determinadas características de determinada população ou fenômeno, valendo-se de técnicas padronizadas para a coleta de dados (GIL, 1999) de maneira a detalhar características de determinado fenômeno, estabelecendo correlações ente variáveis e definindo sua natureza (VERGARA, 1997).

A primeira etapa da pesquisa consistiu na identificação das principais etapas do PDP na indústria processadora de alimentos, definida a partir de uma pesquisa bibliográfica na plataforma Web of Knowledge (a partir dos termos "new product development", "NPD", "innovation", "dairy sector", "research and development", "innovation in the food industry"). A partir dos modelos de PDP propostos por Fuller (1994); Rudolph (1995) e Earle (1997) e a contribuição de especialistas (cinco responsáveis pelo PDP de indústrias processadoras de laticínios no RS, duas de médio e três de grande porte ${ }^{2}$ ) definiu-se um modelo adaptado de PDP a ser avaliado nas etapas seguintes da pesquisa.

Esse modelo de PDP contempla as seguintes fases e etapas: i) na fase de prédesenvolvimento: etapas de análise de mercado, desenvolvimento da estratégia organizacional, geração de idéias, estudo da viabilidade técnica, estudo de marketing, análise financeira, seleção da ideia e conceituação do produto; ii) na fase de desenvolvimento: etapas de identificação do cliente, identificação das necessidades e requisitos do cliente, hierarquização da importância dos itens de qualidade exigidos pelo cliente, conversão dos requisitos dos clientes em especificações do produto, análise e classificação das especificações do produto, análise de viabilidade tecnológica, testes de produto (testes físico-químicos e determinação da formulação), testes de protótipos (definição de ingredientes e embalagens), definição de fornecedores, análise sensorial, produção de lote piloto, elaboração de sistemas de segurança alimentar, determinação da vida de prateleira, definição de registros, planejamento do processo produtivo, plano de gestão do processo de estocagem, plano de gestão do processo de distribuição, produção e lançamento do produto; iii) fase de pós-desenvolvimento: etapas de estudo da eficiência da produção, planejamento do futuro do produto e estudo do comportamento de compras e atitude dos consumidores.

A partir dessa etapa inicial, formulouse uma proposta de questionário estruturado, que avaliava tanto o grau de dificuldade para a realização de cada etapa do PDP (definido na etapa anterior) como a natureza dessa dificuldade (gerencial, financeira, comercial ou tecnológica). Essa proposta de questionário foi submetida a cinco especialistas para avaliação, aperfeiçoamento e adequação do mesmo: dois professores universitários que desenvolvem pesquisas nos temas referentes ao desenvolvimento de produto e pesquisa de mercado no setor de alimentos; um engenheiro de alimentos atuante como analista de P\&D em um centro nacional de pesquisa de uma indústria processadora de laticínios de grande porte; um representante do Sindicato das Indústrias de Laticínios - SINDILAT e; o diretor industrial de uma cooperativa processadora de laticínios de grande porte.

Para avaliar o grau de dificuldade na realização das etapas do PDP nesse questionário, foi proposta uma escala Likert de cinco pontos envolvendo os seguintes parâmetros:

2 Nessa pesquisa, considerou-se, arbitrariamente, como de pequeno porte as indústrias que processam até $20.000 \mathrm{~L} /$ dia de leite, de médio porte as que processam entre 20.000 e $100.000 \mathrm{~L} /$ dia e de grande porte as que processam mais de $100.000 \mathrm{~L} /$ dia. Essa proposta de estratificação não encontra suporte em qualquer pesquisa setorial prévia, porém, nesse caso, teve a validação dos especialistas consultados na primeira etapa da pesquisa. 
dificuldade muito baixa $=1$, dificuldade baixa $=2$, dificuldade média $=3$, dificuldade alta $=$ 4 , dificuldade muito alta $=5$.

Na terceira etapa, buscou-se a validação desse instrumento (o questionário estruturado), realizando um pré-teste do mesmo junto a tomadores de decisão de doze indústrias processadoras de laticínios do RS. O préteste se refere ao teste do instrumento de coleta de dados junto a um pequeno grupo de entrevistados, oriundos da mesma população de pesquisa final, com o objetivo de identificar a clareza das questões. A amostra selecionada foi composta por $10 \%$ da população final da pesquisa (104 empresas) e, sua estratificação, em função do porte, reflete a mesma proporcionalidade da amostra final.

$\mathrm{Na}$ última etapa da pesquisa, o questionário estruturado foi aplicado por meio da internet a partir do envio de seu link para uma lista de gerentes, envolvidos com o processo de PDP, de 104 empresas processadoras de laticínios, de pequeno, médio e grande porte, no estado do RS, registradas nos órgãos de inspeção sanitária: Serviço de Inspeção Federal - SIF e Coordenadoria de Inspeção de Produtos de Origem Animal - CISPOA.

Para coleta das informações e análise dos resultados, foi utilizado o software Sphynx versão 5.0. Os dados (respostas) foram avaliados considerando a distribuição de frequência das respostas e média aritmética.

\section{RESULTADOS E DISCUSSÃO}

O questionário foi respondido por representantes de 68 empresas $^{3}$ entre os dias 15 de setembro e 14 de novembro de 2011. A caracterização das empresas conforme o porte foi de $81 \%$ de pequeno, $16 \%$ de médio e $3 \%$ de grande porte.
Os resultados da pesquisa evidenciaram que a fase do PDP que concentra a maior parte das etapas consideradas como de dificuldade alta ou muito alta (médias acima de 4,0) é a fase de desenvolvimento e, a seguir, a fase de pós-desenvolvimento. Nas etapas do PDP consideradas como de maior dificuldade de realização, predominam as restrições de natureza gerencial (Tabela 1). Esse resultado encontra subsídio nos achados de Lima et al. (2009).

Em particular, a etapa do PDP em que os tomadores de decisão das empresas consultadas encontram maior dificuldade é a "elaboração de testes de protótipos - explorando novos ingredientes e embalagens". Essa etapa apresenta extrema importância já que nela definem-se atributos sensoriais de grande influência na intenção de compra do consumidor final (VIANA; RÉVILLION, 2010).

A segunda etapa do PDP considerada como de maior dificuldade para execução foi a "definição dos fornecedores". Essa pode ser uma etapa crítica para o PDP pois, de acordo com dados publicados pelo IBGE (2010), $76 \%$ das indústrias de fabricação de produtos alimentícios implementaram inovações a partir da interação com seus fornecedores.

As fases que foram consideradas como de menor grau de dificuldade foram relativas ao "estudo de marketing" (por questões financeiras) e a "hierarquização da importância dos itens de qualidade exigidos pelo cliente" (em função de aspectos comerciais) (Tabela 2). Entretanto, esse resultado está em desacordo com dados publicados em pesquisas relacionadas à aplicação de métodos de desenvolvimento de produtos como o $\mathrm{QFD}^{4}$, cujos autores destacam que a hierarquização das necessidades dos clientes é uma fase que apresenta alta dificuldade

3 Para as empresas que possuem filiais, mas têm seu centro de desenvolvimento de produtos unificado (mesmo que fora do RS), optou-se por considerar a resposta somente uma vez, já que o PDP é realizado por uma única equipe para várias filiais. 
(POLIGNANO et al., 2000; CARNEVALLI; MIGUEL, 2008).

Considerando-se todas as etapas do PDP consideradas nessa pesquisa, cerca de $40 \%$ das dificuldades encontradas foram avaliadas como de origem gerencial contra $20 \%$ de ordem financeira, $20 \%$ de natureza comercial e $20 \%$ de natureza tecnológica.

Os resultados dessa pesquisa apresentam, aparentemente, contradições de difícil interpretação considerando-se que: i) se o setor processador de alimentos baseia-se, em grande parte, no apoio de fornecedores de ingredientes, coadjuvantes de tecnologia, aditivos e embalagens para inovar, por que as duas fases de maior dificuldade do PDP estão relacionadas a essa relação? ii) Se o "estudo do comportamento de compras e atitude dos consumidores", na fase de pósdesenvolvimento é um fator restritivo por que o "estudo de marketing" na fase de prédesenvolvimento não o seria, considerando a convergência de habilidades e recursos nas duas fases?

Em relação ao primeiro questionamento, é possível que a natureza tecnológica da dificuldade possa sinalizar uma tendência de realização da etapa "teste de protótipos explorando novos ingredientes e embalagens" intra-muros com pessoal técnico da própria empresa - o que, de fato, representa um desafio muito maior do que a adoção de novas tecnologias (embutidas em insumos e equipamentos) a partir da orientação dos fornecedores. Também é possível que a definição de fornecedores represente uma etapa de grande dificuldade frente à setorização das empresas (setor "administrativo" e setor "da produção") - uma premissa que justificaria a natureza "gerencial" da dificuldade - o que,

Tabela 1 - Etapas do PDP consideradas de maior dificuldade para sua realização

\begin{tabular}{|c|c|c|c|c|}
\hline Fase do PDP* & $\begin{array}{l}\text { Etapa do } \\
\text { PDP }\end{array}$ & $\begin{array}{l}\text { Média dos } \\
\text { resultados }\end{array}$ & $\begin{array}{l}\text { Desvio } \\
\text { padrão }\end{array}$ & $\begin{array}{l}\text { Natureza da } \\
\text { dificuldade }\end{array}$ \\
\hline $\mathrm{D}$ & $\begin{array}{l}\text { Testes de protótipos - } \\
\text { explorando novos } \\
\text { ingredientes e embalagens }\end{array}$ & 4,6 & 0,61 & Tecnológica \\
\hline $\mathrm{D}$ & Definição de fornecedores & 4,4 & 0,79 & Gerencial \\
\hline PÓS & $\begin{array}{l}\text { Estudo do comportamentos } \\
\text { de compras e atitude } \\
\text { dos consumidore }\end{array}$ & 4,3 & 0,90 & Comercial \\
\hline PRÉ & Análise financeira & 4,2 & 1,01 & Financeira \\
\hline $\mathrm{D}$ & $\begin{array}{l}\text { Planejamento do } \\
\text { processo produtivo }\end{array}$ & 4,2 & 0,83 & Gerencial \\
\hline PÓS & $\begin{array}{l}\text { Estudo da eficiência } \\
\text { da produção }\end{array}$ & 4,2 & 0,94 & Gerencial \\
\hline
\end{tabular}

* PRÉ: fase de pré - desenvolvimento; D: fase de desenvolvimento; PÓS: fase de pós-desenvolvimento.

4 O método QFD pode ser considerado um grupo de ferramentas de planejamento que auxiliam na introdução de novos ou melhorados produtos no mercado através do controle do desenvolvimento do processo e do foco na satisfação do consumidor (Costa et al., 2000). 
freqüentemente, leva a conflitos relacionados à percepção da relação de custo/benefício dos insumos utilizados.

Outra explicação plausível repousa no predomínio de empresas de pequeno porte: talvez essas encontrem maior dificuldade na identificação de fornecedores qualificados e no estabelecimento de relações de efetiva cooperação técnica para a escolha e utilização de novos ingredientes e embalagens.

Já a segunda questão é de maior dificuldade no estabelecimento de hipóteses. De fato, toda organização que tiver um setor de marketing bem estruturado deverá se preocupar tanto com as características das necessidades dos consumidores que orientam o desenvolvimento de produtos como com o grau de atendimento dessas necessidades após a compra/consumo do produto. Portanto, fica o questionamento se houve entendimento das dimensões dessas etapas pelos respondentes.

\section{CONCLUSÕES}

As etapas do PDP em que os tomadores de decisão das empresas consultadas encontram maior dificuldade foram relacionadas a "testes de protótipos - explorando novos ingredientes e embalagens" (dificuldade de natureza tecnológica), a "definição de fornecedores" (dificuldade de natureza gerencial), na fase de desenvolvimento, e o "estudo do comportamento de compras e atitude dos consumidores" (dificuldade de natureza comercial), depois do lançamento do novo produto. Considerando-se todas as etapas do PDP contempladas nessa pesquisa, predominam as dificuldades de natureza gerencial.

Essas dificuldades podem estar relacionadas a uma mudança do perfil das inovações desenvolvidas nesse setor, de menor dependência dos fornecedores, buscando uma maior apropriabilidade das mesmas - o que exige uma maior capacitação técnica de seus quadros e uma visão mais alinhada, e menos setorizada, entre os profissionais de "produção" e "gestão" das empresas. De outro lado, o predomínio de respondentes atuando em empresas de pequeno porte também pode estar relacionado à hierarquização dessas etapas como de maior dificuldade.

Os resultados dessa pesquisa são limitados tanto pelo escopo do modelo de PDP proposto aos entrevistados como pela restrição de sua abrangência a um único estado, não podendo ser generalizada para outros setores ou para o setor processador de laticínios no Brasil.

Tabela 2 - Etapas do PDP consideradas de menor dificuldade para sua realização

\begin{tabular}{|c|c|c|c|c|}
\hline Fase* & Etapa do PDP & $\begin{array}{l}\text { Média dos } \\
\text { resultados }\end{array}$ & $\begin{array}{l}\text { Desvio } \\
\text { padrão }\end{array}$ & $\begin{array}{l}\text { Natureza da } \\
\text { dificuldade }\end{array}$ \\
\hline $\mathrm{D}$ & $\begin{array}{l}\text { Elaboração de sistemas } \\
\text { de segurança alimentar }\end{array}$ & 1,7 & 1,52 & Tecnológica \\
\hline $\mathrm{D}$ & $\begin{array}{l}\text { Plano de gestão do } \\
\text { processo de estocagem }\end{array}$ & 1,7 & 1,53 & Gerencial \\
\hline PRÉ & Estudo de marketing & 1,5 & 1,66 & Financeira \\
\hline $\mathrm{D}$ & $\begin{array}{l}\text { Hierarquização da } \\
\text { importância dos itens de } \\
\text { qualidade exigidos pelo cliente }\end{array}$ & 1,2 & 1,30 & Comercial \\
\hline
\end{tabular}

* PRÉ: fase de pré- desenvolvimento; D: fase de desenvolvimento; PÓS: fase de pós-desenvolvimento. 


\section{AGRADECIMENTOS}

Os autores agradecem tanto aos especialistas e gerentes consultados pela contribuição para a realização deste estudo, quanto ao Conselho Nacional de Desenvolvimento Científico e Tecnológico $(\mathrm{CNPq})$ pelo financiamento desta pesquisa.

\section{REFERÊNCIAS}

CABRAL, J. E. O. Determinantes da Propensão para Inovar e da Intensidade Inovativa em Empresas da Indústria de Alimentos do Brasil, Revista de Administração Contemporânea RAC, v. 11, n. 4, p. 87-108, 2007.

CARNEVALLI, J. A.; MIGUEL, P. C. Review, analysis and classification of the literature on QFD-Types of research, difficulties and benefits. International Journal of Production Economics, v. 114, n.2, p. 737- 754, 2008. Disponível em: <http://www.sciencedirect. com/science/article/pii/S0925527308001138>. Acesso em 25 set. 2012.

COSTA, A. I. A.; DEKKER, M.; JONGEN, W. M. F. Quality function deployment in the food industry: a review. Trends in Food Science \& Technology, v.11, n.9-10, p. 306-314, 2000. Disponível em: <http://www.sciencedirect. com/science/article/pii/S0924224401000024>. Acesso em 25 set. 2012. doi: 10.1016/S09242244(01)00002-4.

EARLE, M. D. Changes in the food product development process. Trends in Food Science \& Technology, v.8, n.1, p.19-24.1997. Disponível em: <http://www.sciencedirect.com/science/ article/pii/S0924224496200093>. Acesso em 25 set. 2012. doi: 10.1016/S0924-2244(96)20009-3.

FULLER, G. W. New food product development: from concepts to marketplace. Boca Raton: CRC Press, 1994. 304 p.

GIL, A. C. Métodos e técnicas de pesquisa social. 5ed. São Paulo: Atlas, 1999. 216 p.
INSTITUTO BRASILEIRO DE GEOGRAFIA E ESTATÍSTICA (IBGE). Pesquisa de Inovação Tecnológica 2008. Rio de Janeiro: IBGE, Coordenação de Indústria, 2010. 164 p. Disponível em: <http://www.pintec.ibge.gov.br/ >. Acesso em: 18 jan. 2012.

LIMA, M. S.; RÉVILLION, J. P.; PADULA, A. D. Estratégias competitivas e de desenvolvimento de produtos lácteos funcionais: estudos de caso em empresas agroindustriais da região sul do Brasil. Ciência rural, v. 39 , n. 5, p. $1547-$ 1551, 2009.

POLIGNANO, L. A. C.; DRUMOND, F. B.; CHENG, L. C. Mapa de preferência: uma ponte entre marketing e P\&D. In: $\mathrm{CON}$ GRESSO BRASILEIRO DE GESTÃO DE DESENVOLVIMENTO DE PRODUTO, 2. 2000, São Carlos. Anais...São Carlos: UFSCar, 2000. p. 96-102. 1 CD-ROM.

RUDOLPH, M. J. The food product development process. British Food Journal, v. 97, n. 3, p.3-11, 1995. Disponível em: <http://www. emeraldinsight.com/journals.htm?articleid $=146$ 2983\& show $=$ abstract $>$. Acesso em 25 set. 2012. doi: 10.1108/00070709510081408.

TRAIL, B.; MEULENBERG, M. Innovation in the food industry.Agribusiness, v.18, n.1,p.1-21, 2002.

ULRICH K. T.; EPPINGER, S. D. Product Design and Development. 2.ed. Nova Iorque: McGraw-Hill, 2000. 358p.

VERGARA, S. C. Projetos e relatórios de pesquisa em administração. São Paulo: Atlas, 1997. $104 \mathrm{p}$.

VIANA, J. G. A.; RÉVILLION, J. P. Qualidade percebida pelo consumidor de produtos lácteos: modelo teórico e evidências empíricas. Revista Indústria de Laticínios, v. 15, n.87, p. 72-81, 2010. 


\section{Onde você vê leite a gente vê tecnologia}

Há mais de 45 anos, o Macalé é referência em tecnologia no setor de laticínios.

Uma tradição de qualidade e parcerias sólidas que oferecem sempre os melhores ingredientes

e serviços ao mercado laticinista brasileiro.

Por isso, na hora de produzir com qualidade e inovação, conte com a gente.

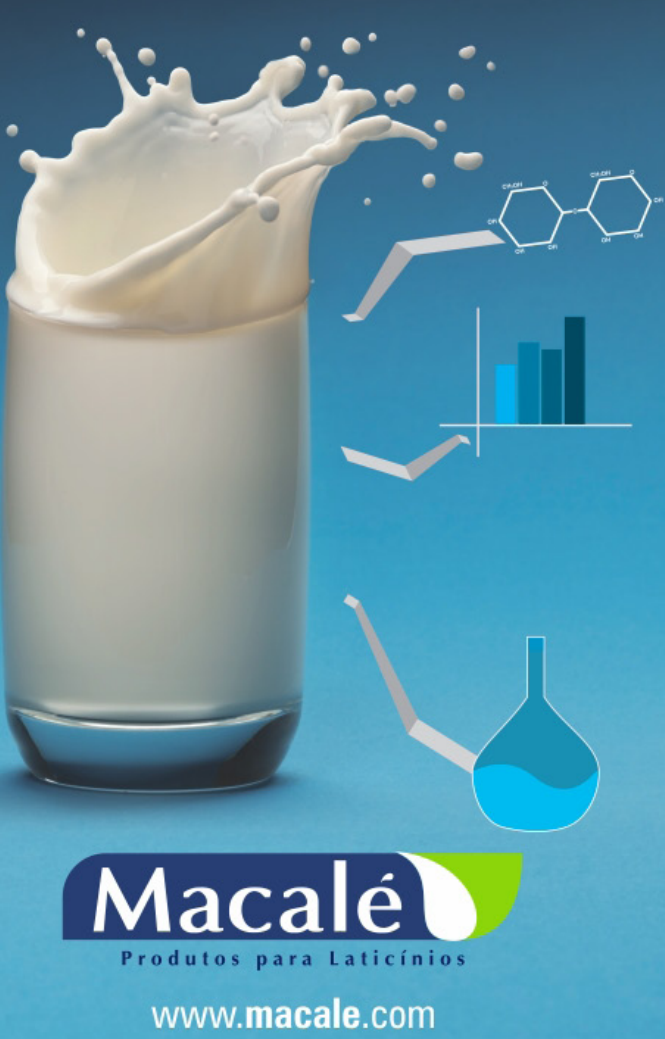

CHR HANSEN

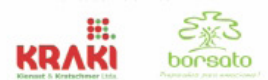

